\title{
Macroecologia de carnívoros do Novo Mundo (Mammalia): envelopes de restrição e análise de padrões filogenéticos
}

\author{
Natália M. Tôrres ${ }^{1,2}$ \& José Alexandre F. Diniz-Filho ${ }^{1}$
}

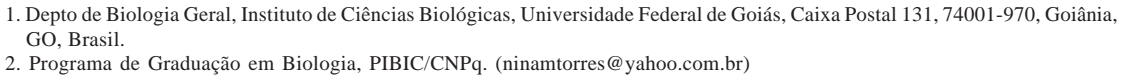

\begin{abstract}
Macroecology of New World carnivores: constraint envelopes and analysis of phylogenetic patterns. The relationship between body size and geographic range was analyzed for 70 species of terrestrial Carnivora ("fissipeds") of the New World, after the control of phylogenetic patterns in the data using phylogenetic eigenvector regression. The analysis from EcoSim software showed that the variables are related as a triangular envelope. Phylogenetic patterns in data were detected by means of phylogenetic correlograms, and 200 simulations of the phenotypic evolution were also performed over the phylogeny. For body size, the simulations suggested a nonlinear relationship for the evolution of this character along the phylogeny. For geographic range size, the correlogram showed no phylogenetic patterns. A phylogenetic eigenvector regression was performed on original data and on data simulated under Ornstein-Uhlenbeck process. Since both characters did not evolve under a simple Brownian motion process, the Type I errors should be around $10 \%$, compatible with other methods to analyze correlated evolution. The significant correlation of the original data $(\mathrm{r}=0.38$; $\mathrm{P}<0.05)$, as well as the triangular envelope, then indicate ecological and adaptive processes connecting the two variables, such as those proposed in minimum viable population models.
\end{abstract}

KEYWORDS. Macroecology, phylogenetic correlograms, eigenvectors, inertia.

\section{INTRODUÇÃO}

Estudos macroecológicos consistem na análise de variáveis ecológicas complexas, tais como tamanho corpóreo, área de distribuição geográfica e densidade populacional, mensuradas em grandes escalas continentais e grandes grupos taxonômicos (BRown \& Maurer, 1989; Brown, 1995). A partir destas análises são derivados modelos empíricos que visam explicar a correlação entre tais variáveis a partir de processos ecológicos em diferentes escalas espaciais e temporais (BROWN \& MAURER, 1989).

Diversas hipóteses têm sido desenvolvidas para explicar a relação positiva entre a área de distribuição geográfica e tamanho corpóreo, tais como a hipótese do tamanho mínimo viável das populações (modelo de Brown \& Maurer) (Brown \& MAurer, 1989; Brown, 1995) e a hipótese das variabilidades ambientais e homeostase funcional (Brown, 1995). No modelo de Brown \& MAURER (1989), essas variáveis não estão relacionadas de modo funcional, havendo sim partes do espaço bivariado que estão ocupadas pelas espécies, definindo um "envelope de restrição" poligonal. Nesse envelope, o limite inferior direito está associado a uma maior probabilidade de extinção de espécies de grande porte que possuem área de distribuição geográfica pequena, já que estas não seriam capazes de manter populações mínimas viáveis em áreas pequenas. Porém, antes de demonstrar a existência desses mecanismos ecológicos e evolutivos, é preciso que se controlem os efeitos filogenéticos e espaciais destas variáveis. Utilizando a análise de regressão por autovetores filogenéticos (PVR, Phylogenetic Eigenvector Regression) (Diniz-FiLHo et al., 1998), pode-se analisar padrões filogenéticos de variação levando em consideração modelos mais complexos de evolução fenotípica, tais como os esperados para esses caracteres ecológicos.

Dentre os modelos estatísticos propostos para avaliar a evolução de caracteres quantitativos, dois têm recebido maior atenção. O movimento Browniano, que tem sido utilizado para modelar a evolução por um processo aleatório de deriva genética (FelsensteIn, 1985, 1988; LyNCH, 1990; MARTINS, 1994), parte do princípio de que a mudança no caráter estudado ocorre com uma taxa constante e não é direcional ao longo do tempo. O processo Ornstein-Uhlenbeck é mais utilizado para modelar a evolução de caracteres complexos controlados por sistemas poligênicos e submetidos a várias pressões seletivas (especialmente seleção estabilizadora) (FELSENSTEIN, 1988; Martins, 1994; Hansen \& Martins, 1996). Neste modelo, as mudanças evolutivas produzidas pela deriva são contrabalançadas por uma força de restrição, interpretada biologicamente como efeito de seleção estabilizadora, que "força" o caráter de volta ao ponto central de variação (o pico adaptativo). Uma vez que os dois processos produzem curvas diferentes da relação entre variância interespecífica e tempo de divergência entre as espécies, eles podem ser distintos em correlogramas filogenéticos (GITTLEMAN \& Kot, 1990; GITTLEMAN et al.,1996). Assim, é possível distinguir entre os processos de divergência interespecífica e utilizá-los para avaliar o desempenho estatístico dos métodos utilizados para correlacionar os caracteres macroecológicos, tais como oPVR.

O objetivo é analisar a relação entre o tamanho do corpo e a área de distribuição geográfica das espécies de mamíferos carnívoros nas Américas, utilizando métodos de aleatorização para a definição de envelopes de 
restrição após o controle dos padrões filogenéticos existentes nas variáveis pelo método do PVR.

\section{MATERIAL E MÉTODOS}

Os dados referentes ao tamanho corpóreo (massa, em kg), bem como as áreas de distribuição geográfica para 70 espécies de carnívoros terrestres, Mammalia, Fissipedia (tab. I), foram obtidos a partir da literatura especializada (BURT \& GROSSENHEIDER, 1980; EISENBERG, 1989; Chapman \& Feldhamer, 1990; Redford \& EISENBERG, 1992, 1999; WILSON \& REEDER, 1993; EMMONS, 1997). As distribuições geográficas dessas espécies foram redesenhadas sobre um mapa da América em projeção de Lambert com área igual, utilizando uma rede com quadrículas de $500 \mathrm{~km}$ de lado cobrindo todo o continente, baseada na utilizada pelo Worldmap (Williams, 2000). A seguir foi feita a contagem do número de quadrículas ocupadas para se determinar a área de distribuição geográfica (extensão de ocorrência) das espécies.

Os valores de massa corpórea e área de distribuição de cada espécie foram transformados em logaritmos naturais e, em seguida, a relação entre estas variáveis foi analisada a fim de determinar se há entre elas uma relação funcional ou um envelope de restrição.

Foram feitas duas análises através de um programa computacional para análises de modelos nulos em ecologia de comunidades chamado EcoSim (GoTELLI \& ENTSMIGER, 2000), a fim de testar a existência de uma relação na forma de um envelope de restrição: a análise de dispersão e a do envelope triangular invertido. Para estes testes, foram realizadas 5000 aleatorizações dos dados originais.

O índice de dispersão é calculado dividindo-se o espaço bivariado em quatro quadrantes, baseado nos pontos medianos das duas variáveis. Este método testa apenas se existe uma agregação das espécies no espaço bidimensional, não assumindo previamente uma "forma" para a relação. O EcoSim conta o número de pontos (espécies) que ocorrem em cada quadrante e a variância entre esses quatro números é uma estimativa do índice de dispersão das espécies nesse espaço bidimensional. Se os dados originais são aleatoriamente distribuídos neste espaço, então a variância observada será similar à distribuição das 5000 variâncias calculadas para o conjunto de dados aleatorizados. Ao contrário, se os pontos são concentrados em um dos quadrantes, a hipótese nula será rejeitada e a variância observada será significativamente maior que a esperada.

Tabela I. Carnívoros analisados, com os seus respectivos valores para a massa corpórea (em kg) e para a área de distribuição geográfica (em número de quadrículas, NQ, de $500 \mathrm{~km}$ de lado).

\begin{tabular}{|c|c|c|c|c|c|}
\hline Espécies & Peso & NQ & Espécies & Peso & NQ \\
\hline Mustela nigripes (Audubon \& Bachman, 1851) & 0,51 & 3,50 & Bassaricyon alleni Thomas, 1880 & 1,25 & 5,75 \\
\hline M. erminea Linnaeus, 1758 & 0,09 & 20,25 & B. beddardi Pocock, 1921 & 1,25 & 1,00 \\
\hline M. frenata Lichtenstein, 1831 & 0,21 & 20,00 & B. gabbii J. A. Allen, 1876 & 1,25 & 0,75 \\
\hline M. nivalis Linnaeus, 1766 & 0,04 & 15,75 & B. lasius Harris, 1932 & 1,58 & 0,13 \\
\hline M. africana Desmarest, 1818 & 0,22 & 5,25 & B. pauli Enders, 1936 & 1,58 & 0,13 \\
\hline M. felipei Izor \& de la Torre, 1978 & 0,38 & 0,25 & Potos flavus (Schreber, 1774) & 2,99 & 14,50 \\
\hline M. vison Schreber, 1777 & 1,07 & 21,50 & Ursus arctos Linnaeus, 1758 & 266,50 & 16,75 \\
\hline Martes americana (Turton, 1806) & 0,63 & 16,75 & U. americanus Pallas, 1780 & 77,50 & 17,75 \\
\hline M. pennanti (Erxleben, 1777) & 3,37 & 5,50 & Tremarctos ornatus (F. G. Cuvier, 1825) & 199,98 & 1,50 \\
\hline Gulo gulo (Linnaeus, 1758) & 20,75 & 15,00 & Canis lupus Linnaeus, 1758 & 43,25 & 27,70 \\
\hline Eira barbara (Linnaeus, 1758) & 3,90 & 20,00 & C. latrans Say, 1823 & 12,75 & 27,00 \\
\hline Galictis cuja (Molina, 1782) & 1,60 & 5,50 & Pseudalopex culpaeus (Molina, 1782) & 7,39 & 2,00 \\
\hline G. vittata (Schreber, 1776) & 3,20 & 17,75 & P. griséus (Gray, 1837) & 3,90 & 3,75 \\
\hline Lyncodon patagonicus (Blainville, 1842) & 1,05 & 0,75 & P. gymnocercus $\quad$ (G. Fischer, 1814) & 4,75 & 1,00 \\
\hline Taxidea taxus (Schreber, 1777) & 7,65 & 12,25 & P. sechurae (Thomas, 1900) & 2,19 & 0,25 \\
\hline Lontra provocax (Thomas, 1908) & 4,21 & 1,75 & P. vetulus (Lund, 1842) & 2,75 & 2,25 \\
\hline L. longicaudis (Olfers, 1818) & 5,80 & 18,75 & Atelocynus microtis (Sclater, 1883) & 7,00 & 6,00 \\
\hline L. canadensis (Schreber, 1777) & 9,35 & 17,00 & Cerdocyon thous (Linnaeus, 1766) & 5,80 & 15,50 \\
\hline Pteronura brasiliensis (Gmelin, 1788) & 26,50 & 14,50 & Chrysocyon brachyurus (Illiger, 1815) & 23,74 & 5,50 \\
\hline Conepatus chinga (Molina, 1782) & 1,85 & 3,50 & Speothos venaticus (Lund, 1842) & 6,00 & 16,50 \\
\hline C. leuconotus (Lichtenstein, 1832) & 1,80 & 0,50 & Vulpes vulpes (Linnaeus, 1758) & 5,60 & 27,00 \\
\hline C. mesoleucus (Lichtenstein, 1832) & 2,80 & 3,00 & V. velox (Say, 1823) & 3,00 & 4,00 \\
\hline C. humboldtii Gray, 1837 & 0,54 & 1,25 & Urocyon cinereoargenteus (Schreber, 1775) & 3,69 & 14,25 \\
\hline C. semistriatus (Boddaert, 1785) & 3,99 & 4,50 & Panthera onca (Linnaeus, 1758) & 90,03 & 25,25 \\
\hline Mephitis macroura Lichtenstein, 1832 & 0,96 & 3,50 & Lynx canadensis Kerr, 1792 & 10,10 & 15,50 \\
\hline M. mephitis (Schreber, 1776) & 2,30 & 19,75 & L. rufus (Schreber, 1777) & 11,20 & 13,50 \\
\hline Spilogale putorius (Linnaeus, 1758) & 0,57 & 6,50 & Leopardus tigrinus (Schreber, 1775) & 2,19 & 20,25 \\
\hline S. pygmaea Thomas, 1898 & 0,57 & 0,25 & Oncifelis geoffroyi (Orbigny \& Gervais, 1844) & 3,80 & 4,75 \\
\hline Procyon cancrivorus (G. Cuvier, 1798) & 8,80 & 21,00 & O. guigna (Molina, 1782) & 2,23 & 1,25 \\
\hline P. lotor (Linnaeus, 1758) & 6,30 & 17,00 & O. colocolo (Molina, 1782) & 2,99 & 6,25 \\
\hline Nasua narica (Linnaeus, 1766) & 8,81 & 4,50 & Oreailurus jacobita (Cornalia, 1865) & 3,99 & 1,75 \\
\hline N. nasua (Linnaeus, 1766) & 4,05 & 19,75 & Leopardus pardalis (Linnaeus, 1758) & 7,90 & 24,50 \\
\hline Nasuella olivacea (Gray, 1865) & 1,38 & 0,50 & L. wiedii (Schinz, 1821) & 3,20 & 21,75 \\
\hline Bassariscus astutus (Lichtenstein, 1830) & 0,92 & 6,00 & Herpailurus yaguaroundi (Lacépède, 1809) & 2,59 & 24,00 \\
\hline B. sumichrasti (Saussure, 1860) & 1,05 & 1,25 & Puma concolor (Linnaeus, 1771) & 37,01 & 36,00 \\
\hline
\end{tabular}


O envelope triangular invertido é definido operacionalmente pelo EcoSim com base nos pontos máximos, mínimos e medianos das duas variáveis, permitindo assim um teste do modelo de Brown \& Maurer (Brown \& Maurer, 1989). Em seguida, o número de espécies que está dentro desse envelope é registrado e após à aleatorização das variáveis originais, esse número observado é comparado à distribuição nula gerada pelas simulações. Se o envelope realmente existe nos dados observados, o número de espécies situadas dentro dele deve ser significativamente maior que a distribuição de números no envelope obtida após os procedimentos de aleatorização.

Utilizou-se para as análises comparativas uma parte da filogenia composta (supertree) de Carnivora (Mammalia) recentemente elaborada por BININDA-EMONDS et al. (1999), incluindo apenas as 70 espécies de carnívoros do Novo Mundo (fig. 1). Esta filogenia composta é construída utilizando-se simultaneamente todas as informações disponíveis para o grupo, com base em dados morfológicos, comportamentais, ecológicos e moleculares, sendo os tempos de divergência entre as linhagens estimados tanto com base no registro fóssil quanto em modelos de evolução molecular. No caso, o tempo máximo de divergência entre as espécies consideradas neste estudo é de 53,8 milhões de anos.

A partir desta filogenia, os padrões filogenéticos no tamanho corpóreo e na área de distribuição geográfica foram detectados através de correlogramas filogenéticos (Gittleman \& Kot, 1990; Gittleman et al.,1996), que expressam as variações na semelhança entre pares de espécies em função do tempo de divergência entre elas. Os índices I de Moran, que são interpretados como coeficientes de autocorrelação (DINIZ-FILHO, 2000, 2001), foram utilizados para definir esta semelhança em 5 classes de tempo (em milhões de anos) ao longo da filogenia: 0,010,76; 10,76-21,52; 21,52-32,28; 32,28-43,04; 43,04-53,8.

Através da rotina PDSIMUL do programa PDAP
(Phenotypic Diversity Analysis Program) (GARLAND et al., 1993) foram realizadas 200 simulações da evolução fenotípica sobre a filogenia, a fim de avaliar como os processos de movimento Browniano e OrnsteinUhlenbeck (O-U) aparecem nos correlogramas filogenéticos. O PDSIMUL parte do valor médio do caráter, na raiz da filogenia, e gera a evolução desse caráter através do modelo de evolução escolhido (movimento Browniano e processo O-U). No movimento Browniano, a variância da mudança em cada instante de tempo é diretamente proporcional à variância fenotípica nos taxa terminais, e passa a ocorrer independentemente em cada linhagem após os eventos de especiação (definidos pela topologia da filogenia-base utilizada), não havendo restrições para a direção e magnitude relativa das mudanças em tempo contínuo (simulando assim um efeito de deriva genética). Já no processo O-U, as mudanças evolutivas produzidas pela deriva são contrabalançadas por uma força de restrição, que "força" o caráter de volta ao ponto central de variação, análoga ao processo biológico de seleção estabilizadora (DINIZ-FILHO, 2000). Para as simulações realizadas neste trabalho, foram utilizados sete valores para a força de restrição da seleção estabilizadora $(\alpha)$, variando de zero (equivalente ao movimento Browniano) até $\alpha=12 / h$, onde $h$ é o comprimento total da filogenia. As simulações foram então analisadas através de correlogramas filogenéticos, construídos utilizando as mesmas classes definidas para a análise dos dados observados, através do programa AUTOPHY, escrito em linguagem Basic (DinIZ-FILHO, 2001).

Como os correlogramas detectaram um padrão filogenético significativo para a massa corpórea em carnívoros da América, é de interesse também caracterizar o quanto da variação total do caráter pode ser atribuída à filogenia (inércia filogenética). Foi realizada então uma análise de regressão por autovetores filogenéticos (Phylogenetic Eigenvector Regression, PVR) (DINIz-FILHO, 2000), método no qual a idéia básica é calcular uma matriz

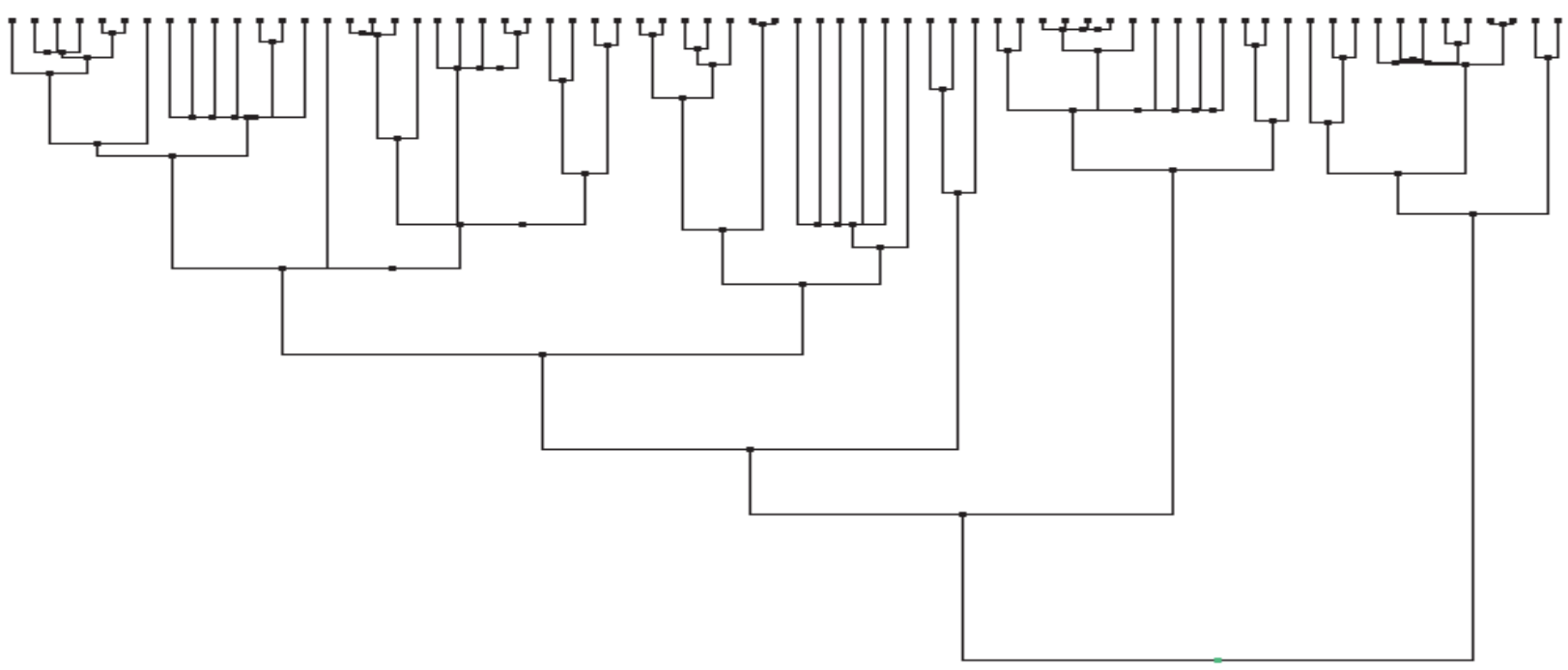

Fig. 1. Filogenia das 70 espécies de carnívoros analisados. As espécies, da esquerda para a direita, seguem a ordem apresentada na tab. I. 
de distâncias filogenéticas entre as espécies, com base na filogenia pré-estabelecida, e extrair os autovalores e autovetores dessa matriz utilizando uma análise de coordenadas principais. Isso transforma a representação das relações entre as espécies de uma "árvore" em um conjunto de vetores, que podem ser utilizados como variáveis preditoras (x) em uma regressão múltipla (SoKAL \& RohlF, 1995) na qual o caráter de interesse é a variável resposta (y). Desta forma, o coeficiente de determinação $\left(\mathrm{R}^{2}\right)$ é utilizado como estimador da inércia filogenética, enquanto que a correlação entre os resíduos de modelos ajustados para diferentes caracteres estima a correlação entre eles independente dos padrões filogenéticos (DINIZFILHo et al.,1998).

Neste trabalho, o PVR foi aplicado para os dados reais e para as simulações por processo O-U previamente descritas, utilizando um número crescente de autovetores (eixos), obtidos através do programa NTSYS-Pc (RoHLF, 1989), e que representam uma parte cada vez maior da filogenia. Como, sob a hipótese nula, a evolução entre o tamanho corpóreo e a área de distribuição foi considerada independente, a correlação intrínseca entre os dois processos de evolução, também denominada "correlação de input" (MARTINS \& GARLAND, 1991), foi estabelecida como zero nas simulações. Essa correlação intrínseca é dada pela correlação entre os caracteres em termos dos eventos de mudança em cada um deles em cada instante de tempo, ao longo do processo evolutivo, e que, associada a outros fatores, explica a correlação entre os caracteres ao longo das espécies. Assim, é possível calcular a probabilidade exata de erro Tipo I do método sob as condições desejadas, levando-se em consideração tanto os diferentes modelos de evolução quanto as diferentes estruturas filogenéticas (autovetores) no modelo do PVR, para este conjunto de dados específico. Essa probabilidade de erro Tipo I foi estimada contandose o número de correlações "significativas" a um dado nível de significância (5\%) quando, na verdade, a correlação paramétrica foi de fato zero.

\section{RESULTADOS E DISCUSSÃO}

A correlação linear entre as duas variáveis foi positiva e significativa $(\mathrm{r}=0,302 ; \mathrm{P}=0,011)$. A análise de dispersão para os dados mostrou que a variação observada entre os pontos nos quatro quadrantes (igual a 40,33) dificilmente seria obtida por acaso $(\mathrm{P}=0,009 \mathrm{com}$ base em 5000 aleatorizações). Observou-se, também, que das 70 espécies estudadas, 63 encontram-se dentro do envelope de restrição triangular invertido definido pelo EcoSim (fig. 2). As aleatorizações permitem afirmar que existe uma probabilidade muito pequena $(P=0,0032)$ de que as espécies tenham esta disposição triangular ao acaso.

O padrão filogenético no tamanho do corpo está estruturado de forma contínua (fig. 3), em um gradiente temporal, indicando que até uma dada distância filogenética (cerca de 30 milhões de anos), as espécies são relativamente semelhantes entre si. Para a área de distribuição geográfica, entretanto, não foi detectado nenhum padrão filogenético, com todos os valores de I de Moran próximos ao esperado pela hipótese nula (ou seja, ausência de padrão fillogenético no caráter).
Comparando os correlogramas de tamanho do corpo e da área de distribuição com os perfis simulados pelo PDSIMUL (fig. 4), observou-se que, para o tamanho do corpo, as simulações com $\alpha=2$ foram as mais próximas do observado, indicando que existe uma pequena nãolinearidade para a evolução deste caráter ao longo da filogenia. Para a área de distribuição geográfica, o perfil do correlograma foi compatível com os obtidos através de valores muito elevados de $\alpha(\alpha=12)$, ou seja, compatível com correlogramas sem padrão filogenético.
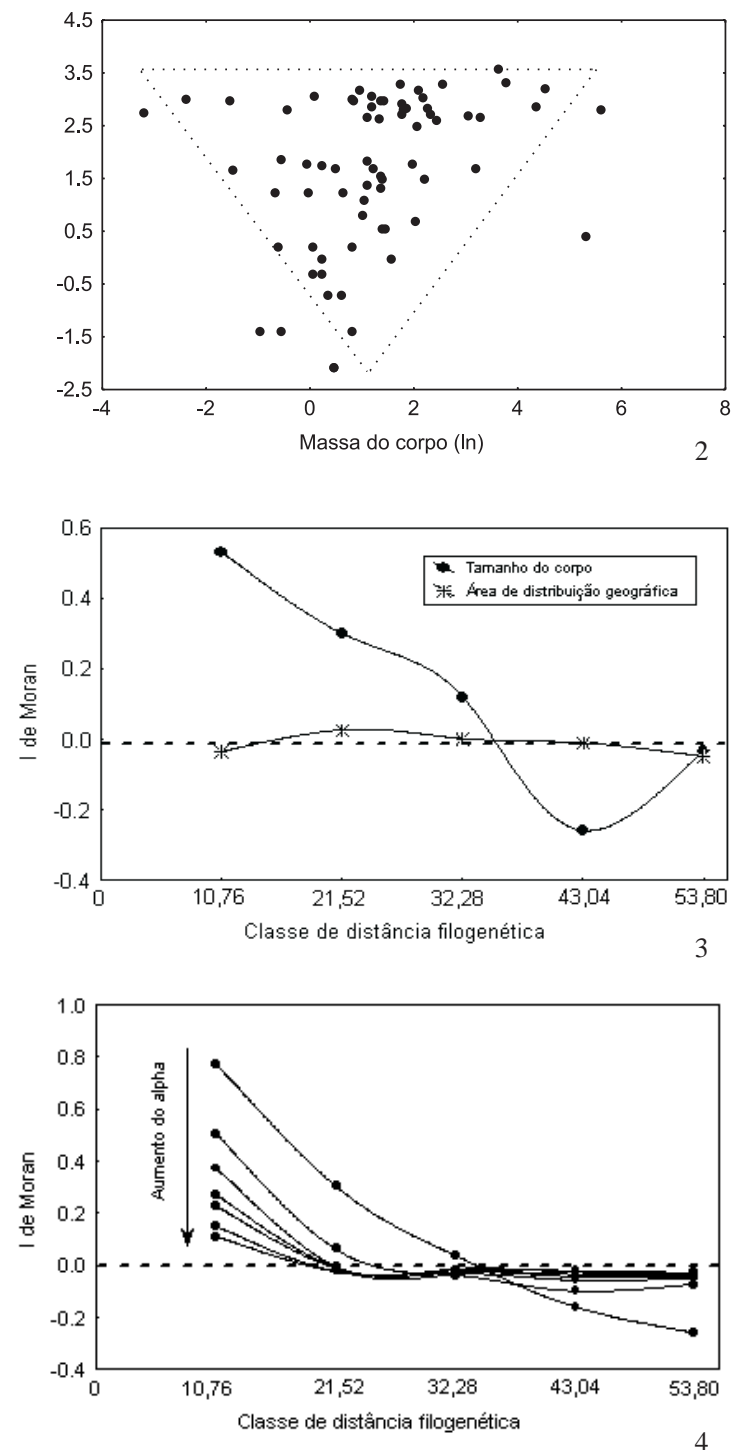

Figs. 2-4. 2, Relação entre área de distribuição geográfica e massa corpórea para as 70 espécies de carnívoros da América. A linha tracejada indica o envelope de restrição triangular definido operacionalmente pelo EcoSim com base nos pontos máximos, mínimos e medianos das duas variáveis; 3, correlogramas filogenéticos para os dados reais do peso do corpo e área de distribuição das 70 espécies de carnívoros. A linha tracejada indica o valor do I de Moran esperado sob a hipótese nula de ausência de autocorrelação. As classes de distância filogenética são os intervalos de tempo em milhões de anos; 4, correlogramas filogenéticos simulados sobre a filogenia das 70 espécies de carnívoros da América, utilizando valores crescentes de $\alpha$. A linha tracejada indica o valor do I de Moran esperado sob a hipótese nula de ausência de autocorrelação filogenética. As classes de distância filogenética são os intervalos de tempo em milhões de anos. 
Através do PVR, observa-se que a inércia filogenética, estimada através dos coeficientes de determinação $\left(\mathrm{R}^{2}\right)$, foi significativamente maior para o tamanho corpóreo do que para a área de distribuição (fig. 5). Utilizando cinco autovetores no PVR, por exemplo, os $\mathrm{R}^{2}$ para o tamanho do corpo e para a distribuição geográfica foram iguais a 0,50 e 0,11 , respectivamente. Esses valores foram, portanto, compatíveis com os perfis observados nos correlogramas. Entretanto, eles variaram também de acordo com o número de autovetores utilizados no modelo (tab. II).

Tabela II. Resultados de regressão por autovetores filogenéticos para os dados reais, considerando diferentes números de eixos extraídos, incluindo as inércias filogenéticas para os dois caracteres analisados $\left(R^{2}\right)$, o teste de sua significância pela estatística $F$, a correlação entre os resíduos do modelo (r) e o teste t para sua significância.

\begin{tabular}{lrrrrrrrrrr}
\hline & \multicolumn{11}{c}{ Eixos } \\
\hline & 1 & 2 & 3 & 4 & 5 & 6 & 7 & 8 & 9 & 10 \\
$\mathrm{R}_{\text {tam }}^{2}$ & 0,09 & 0,17 & 0,21 & 0,47 & 0,50 & 0,52 & 0,54 & 0,58 & 0,60 & 0,61 \\
$\mathrm{R}_{\text {arraa }}^{2}$ & 0,05 & 0,05 & 0,08 & 0,09 & 0,11 & 0,19 & 0,22 & 0,23 & 0,25 & 0,26 \\
$\mathrm{~F}_{\mathrm{A}}$ & 6,84 & 6,66 & 5,84 & 14,32 & 12,82 & 11,37 & 10,18 & 10,39 & 9,85 & 9,25 \\
$\mathrm{~F}_{\mathrm{B}}$ & 3,61 & 1,78 & 1,99 & 1,61 & 1,56 & 2,47 & 2,49 & 2,24 & 2,28 & 2,02 \\
$\mathrm{r}$ & 0,25 & 0,26 & 0,32 & 0,33 & 0,38 & 0,34 & 0,32 & 0,31 & 0,28 & 0,28 \\
$\mathrm{t}$ & 2,13 & 2,21 & 2,72 & 2,79 & 3,26 & 2,87 & 2,64 & 2,50 & 2,22 & 2,19 \\
\hline
\end{tabular}

$\mathrm{O}$ valor de $t$ associado à correlação do PVR, estimando a correlação independente do efeito filogenético entre as duas variáveis para os dados reais, foi maior que o valor crítico no nível de 5\%, independentemente do número de autovetores utilizados no PVR. Assim, existe uma correlação significativa entre as duas variáveis mesmo quando a estrutura filogenética foi levada em consideração. $\mathrm{O}$ maior valor de correlação foi encontrado para cinco autovetores $(\mathrm{r}=0,38 ; \mathrm{t}=3,261 ; \mathrm{P}<0,01)$ (fig. 6), indicando assim que este é o número de autovetores que permite o controle estatístico de mais informação filogenética que pode levar a interpretações espúrias da correlação entre as variáveis, e, portanto, o que deve ser utilizado para o controle dos padrões de modo mais conservativo.

Quando são utilizados cinco autovetores no PVR, o valor de $\mathrm{R}^{2}$ médio, obtido com os dados simulados com evolução por movimento Browniano, foi igual a 0,62 , um pouco maior do que o obtido para os dados reais de tamanho do corpo. Esse resultado é também compatível com a comparação entre os correlogramas reais e simulados, que mostram um padrão com menor estrutura filogenética (não-linearidade) para esta variável.

A probabilidade de erro Tipo I do PVR, calculada a partir das simulações, variou em função do modelo de evolução e do número de autovetores utilizados. Para cinco autovetores, o erro Tipo I no nível de 5\% variou entre 0,22 (movimento Browniano) e 0,078 (O-U com $\alpha=10$ ) (fig. 7). Como os dois caracteres evoluem de modo diferente, a probabilidade de erro Tipo I nos dados reais deve estar em torno de 10-15\%.

Os dois métodos aplicados através do programa EcoSim mostram que a probabilidade de que as variáveis analisadas (tamanho corpóreo e área de distribuição geográfica) sejam independentes uma da outra é praticamente nula para as 70 espécies de Carnivora do Novo Mundo. Como esperado, foi comprovada a existência de um envelope de restrição de formato triangular, de acordo com o modelo de Brown \& Maurer (BRown \& MAURER, 1989; TAYLOR \& Gotelli, 1994). Há, além disso, uma correlação positiva e significativa entre as variáveis. Entretanto, para entender-se qual é a relação real entre estas duas variáveis, precisa-se controlar os efeitos filogenéticos.
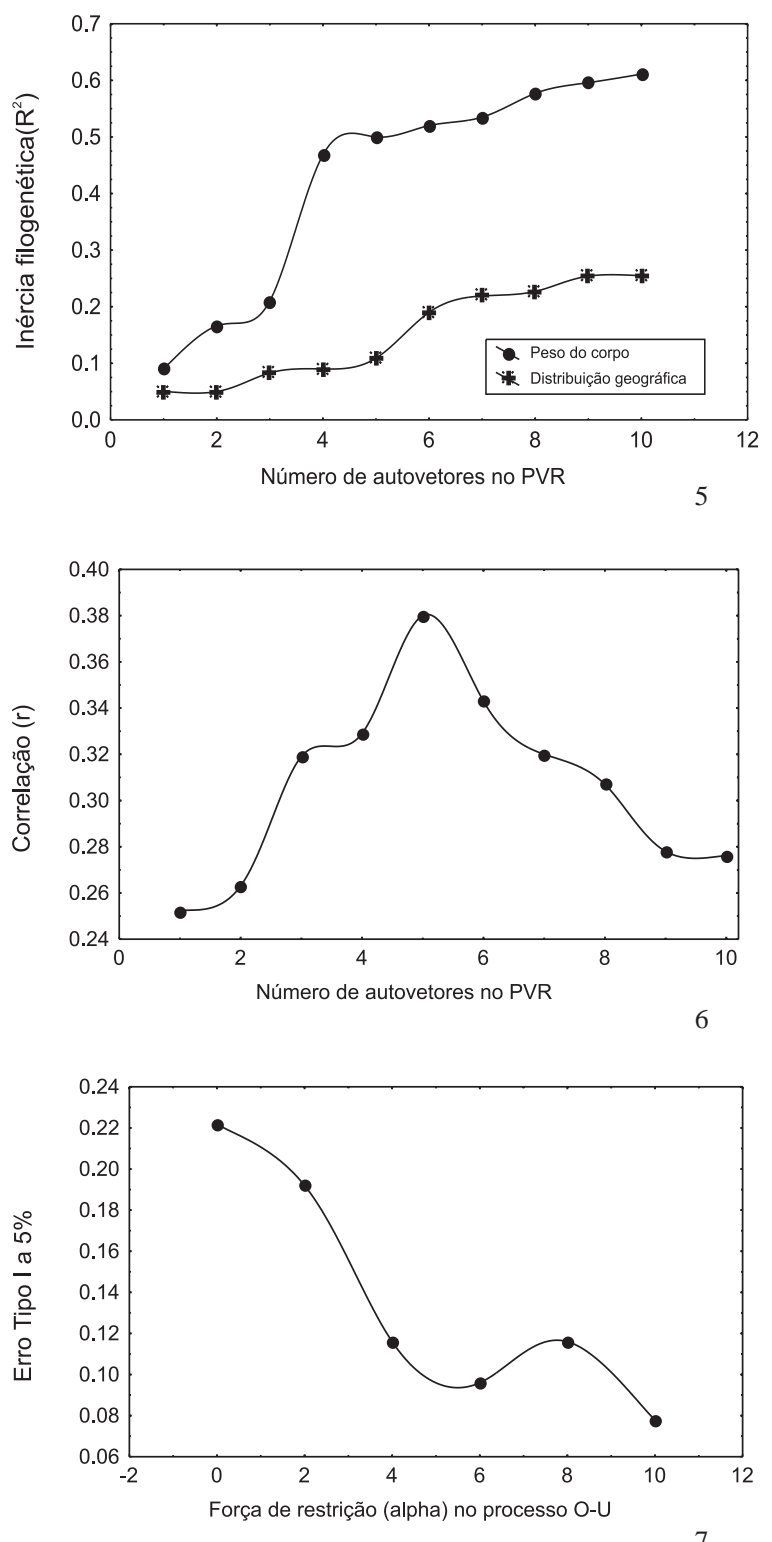

7

Figs. 5-7. 5, Inércia filogenética observada $\left(\mathrm{R}^{2}\right)$ para o tamanho do corpo e área de distribuição geográfica das 70 espécies de carnívoros, em função do aumento no número de eixos da regressão por autovetores filogenéticos; 6 , correlação entre área de distribuição geográfica e tamanho do corpo para as 70 espécies de carnívoros, utilizando números crescentes de autovetores na regressão por autovetores filogenéticos; 7, erro Tipo I no nível de $5 \%$ da regressão por autovetores filogenéticos, para cinco autovetores, em função do aumento da força de restrição da seleção estabilizadora $(\alpha)$ no processo O-U. 
Ao contrário do que ocorre com a área de distribuição geográfica, o tamanho do corpo está freqüentemente estruturado ao longo da filogenia, um padrão que tem sido encontrado para muitos organismos (Cheverud et al.,1985; Gittleman \& Kot, 1990; Edwards \& Kot, 1995; GefFen et al.,1996; GitTLEMAN et al.,1996). Através do correlograma para os dados de tamanho do corpo, pode-se inferir que, a partir da distância temporal de cerca de 30 milhões de anos, não é possível, conhecendo o valor deste caráter em uma espécie, predizer qual será o seu valor em outras espécies. Para os dados de área de distribuição geográfica, nenhum dos índices I de Moran do correlograma foi significativo, indicando assim que não existe um padrão filogenético.

O padrão de evolução significativo ocorre provavelmente porque o tamanho do corpo está sujeito a muitas restrições evolutivas, estando correlacionado com muitas variáveis ecológicas e fisiológicas. A ausência de padrão para a área de distribuição geográfica deve ser função da grande plasticidade ecológica deste caráter ao longo da evolução do grupo, em função de sua rápida resposta a efeitos ambientais, independente de restrições estruturadas filogeneticamente.

Para as análises das simulações, o sinal filogenético nos dados foi maior quando a evolução fenotípica foi modelada por um processo de movimento Browniano, e conforme se aumentou a força de restrição no processo $\mathrm{O}-\mathrm{U}$, a inércia decresceu, ou seja, o padrão filogenético tendeu a desaparecer com o aumento da pressão de seleção estabilizadora, tanto nos correlogramas quanto na inércia filogenética $\left(\mathrm{R}^{2}\right)$ medida pelo PVR. Como os correlogramas e o $\mathrm{R}^{2}$ do PVR mostram que os dois caracteres não devem ter evoluído por movimento Browniano, havendo uma pequena restrição no processo $\mathrm{O}-\mathrm{U}$ para o tamanho corpóreo e uma grande restrição para a área de distribuição geográfica, o erro Tipo I deve estar em torno de $10 \%-15 \%$ para a estimativa de correlação real entre os caracteres, o que é compatível com outros métodos para avaliar a correlação evolutiva (DINızFILHo, 2000).

O PVR não sobreestima muito o erro Tipo I da correlação entre as duas variáveis e, como todos os valores de correlação estimados pelo PVR são bastante significativos (tab. II), a correlação não deve ser de fato um artefato filogenético. É importante notar que essa correlação está subestimada (de modo que a análise tende a ser conservativa), já que o EcoSim mostrou previamente que a relação entre as variáveis é melhor descrita pelo envelope de restrição triangular. Assim, deve haver de fato uma correlação adaptativa entre as duas variáveis analisadas nos Carnivora do Novo Mundo, provavelmente seguindo o modelo de populações mínimas de Brown \& Maurer (BROwn \& Maurer, 1989). Segundo este modelo, a forma triangular invertida da relação entre área de distribuição geográfica e tamanho corpóreo é conseqüência, principalmente em termos da improbabilidade de ocupação de espécies no quadrante inferior esquerdo, de extinções diferenciais ao longo do tempo evolutivo, em um processo de dinâmica diferencial de espécies
(Diniz-Filho, 2004). No caso, espécies de grande porte que ocupam áreas de distribuição geográfica relativamente pequenas tenderiam a ser extintas mais freqüentemente, já que estas exigem maiores áreas de vida e, portanto, devem ocorrer em baixas densidades locais. A única maneira de evitar a extinção seria ocupar maiores áreas de distribuição geográfica após a redução da densidade local.

Agradecimentos. A Luís Maurício Bini (Universidade Federal de Goiás) pelas valiosas discussões sobre macroecologia e métodos filogenéticos comparativos. Ao CNPq, CAPES, FUNAPE/PRPPG, UFG, pelo apoio ao programa de pesquisas em Ecologia e Evolução.

\section{REFERÊNCIAS BIBLIOGRÁFICAS}

Bininda-Emonds, O. R. P.; Gittleman, J. L. \& Purvis, A. 1999. Building large trees by combining phylogenetic information: a complete phylogeny of the extant Carnivora (Mammalia). Biological Reviews, London, 74:143-175.

Brown, J. H. 1995. Macroecology. Chicago, University of Chicago. 269p.

Brown, J. H. \& Maurer, B. A. 1989. Macroecology: the division of food and space among species on continents. Science, Washington, 243:1145-1150.

Burt, W. H. \& Grossenheider, R. P. 1980. A field guide to the mammals. 3.ed. Boston, Houghton Mifflin. 289p.

Chapman, J. A. \& Feldhamer, G. A. 1990. Wild mammals of North America. Baltimore, The Johns Hopkins University. $1147 \mathrm{p}$.

Cheverud, J. M.; Dow, M. M. \& Leutenegger, W. 1985. The quantitative assessment of phylogenetic constraints in comparative analysis: sexual dimorphism in body weight among primates. Evolution, Lawrence, 39:1335-1351.

Diniz-Filho, J. A. F. 2000. Métodos filogenéticos comparativos. Ribeirão Preto, Holos. 162p.

2001. Phylogenetic autocorrelation under distinct evolutionary processes. Evolution, Lawrence, 55:1104-1109. 2004. Macroecology and the hierarchical expansion of evolutionary theory. Global Ecology and Biogeography, Oxford, 13(1):1-5.

Diniz-Filho, J. A. F.; Sant'Ana, C. E. R. \& Bini, L. M. 1998. An eigenvector method for estimating phylogenetic inertia. Evolution, Lawrence, 52:1247-1262.

Edwards, S. V. \& Kот, M. 1995. Comparative methods at the species level: geographic variation in morphology and group size in Grey-Crowned Babblers (Pomatostomus temporalis). Evolution, Lawrence, 49:1134-1146.

Eisenberg, J. F. 1989. Mammals of the neotropics. Chicago, University of Chicago. v.1, 449p.

Emmons, L. H. 1997. Neotropical rainforest mammals: a field guide. 2.ed. Chicago, University of Chicago. 323p.

Felsenstein, J. 1985. Phylogenies and the comparative method. American Naturalist, Chicago, 125:1-15.

1988. Phylogenies and quantitative characters. Annual Review of Ecology and Systematics, Palo Alto, 19:445471 .

Garland, T., Jr.; Dickerman, A. W. et al. 1993. Phylogenetic analysis of covariance by computer simulation. Systematic Biology, London, 42:265-292.

Geffen, E.; Gomprer, M. E. \& Gittleman, J. L. 1996. Size, lifehistory traits, and social organization in the Canidae: a reevaluation. American Naturalist, Chicago, 147:140160.

Gittleman, J. L.; Anderson, C. G. et al. 1996. Phylogenetic lability and rates of evolution: a comparison of behavioral, morphological and life-history traits. In: Martins, E. P. ed. Phylogenies and the comparative method in animal behavior. Oxford, Oxford University. p.166-205.

Gittleman, J. L. \& Kot, M. 1990. Adaptation: statistics and a null model for estimating phylogenetic effects. Systematic Zoology, Washington, 39:227-241. 
Gotelli, N. J. \& Entsmiger, G. L. 2000. Ecosim: null models software for ecology. Version 5.0. Acquired Intelligence \& Kesey-Bear. <http://homepages.together.net/ gentsmin/ ecosim.htm> março 2002.

Hansen, T. F. \& Martins, E. P. 1996. Translating between microevolutionary process and macroevolutionary patterns: the correlation structure of interspecific data. Evolution, Lawrence, 50:1404-1417.

LyNCH, M. 1990. The rate of morphological evolution in mammals from the standpoint of neutral expectation. American Naturalist, Chicago, 136:727-741.

Martins, E. P. 1994. Estimating the rate of phenotypic evolution from comparative data. American Naturalist, Chicago, 144:193-209.

Martins, E. P. \& Garland, T., JR. 1991. Phylogenetic analysis of the correlated evolution of continuous characters: a simulation study. Evolution, Lawrence, 45:534-557.
Redford, K. H. \& Eisenberg, J. F. 1992. Mammals of the neotropics. Chicago, University of Chicago. v.2, 430p. 1999. Mammals of the neotropics. Chicago, University of Chicago. v.3, 609p.

Rohlf, F. J. 1989. NTSYS: numerical taxonomy and multivariate analysis system. New York, Exeter. 200p.

Sokal, R. R. \& Rohlf, F. J. 1995. Biometry. 3.ed. New York, W. H. Freeman. $887 \mathrm{p}$.

TAYlor, C. M. \& Gotelli, N. J. 1994. The macroecology of Cyprinella: correlates of phylogeny, body size and geographic range. American Naturalist, Chicago, 144:549-569.

Williams, P. 2000. Worldmap IV. London. <www.nhm.ac.uk/ scince/projects/worldmap> março 2002.

Wilson, D. E. \& Reeder, D. M. 1993. Mammal species of the world: a taxonomic and geographic reference. 2.ed. Washington, Smithsonian Institution. 1207p. 